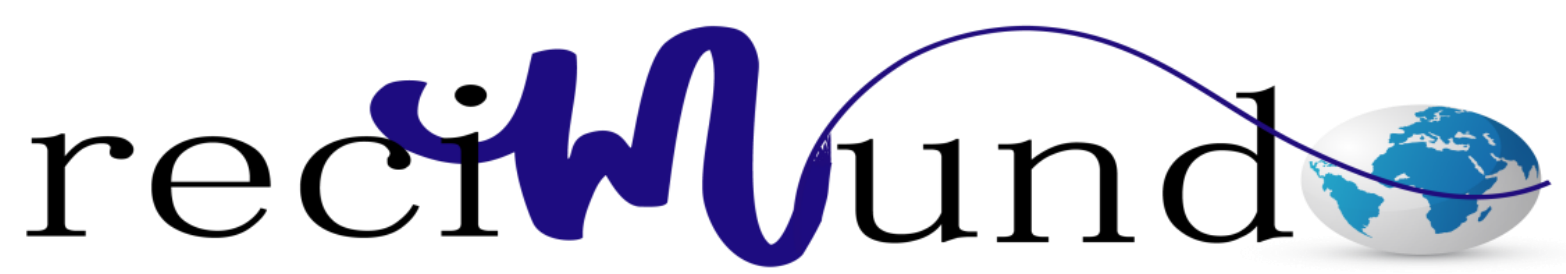

Revista Cientifica Mundo de la Investigación y el Conocimiento

Paulina Elizabeth Duran Mora a ${ }^{\text {; }}$ Hernán David Toro Barrera ${ }^{\text {b; }}$ Mónica Carolina

Calderón Moreira ${ }^{c}$; Diana Galid San Martín Ajila d;

Fernanda Gisell García Reyes ${ }^{e}$; Hugo Javier Saltos Giler ${ }^{\mathrm{f}}$

Tratamiento de luxación en pacientes con problemas óseos

Treatment of dislocation in patients with bone problems

Revista Científica Mundo de la Investigación y el Conocimiento. Vol. 3 núm.2,

abril, ISSN: 2588-073X, 2019, pp. 1214-1247

DOI: 10.26820/recimundo/3.(2).abril.2019.1214-1247

URL: http://recimundo.com/index.php/es/article/view/498

Código UNESCO: 3205 Medicina Interna

Tipo de Investigación: Artículo de Revisión

Editorial Saberes del Conocimiento

Recibido: 15/01/2019

Aceptado: 18/02/2019

Publicado: 30/04/2019

Correspondencia: paupau_edm@hotmail.com

a. Hospital General Privado Duran; Cirugía Plástica; Médico Residente; Ambato, Ecuador; paupau_edm@ @otmail.com

b. Médico Residente de UCI Clínica Centeno; Médico Residente de Emergencia y Jefe de Guardia Hospital General IESS; Médico Cirujano; Manta, Ecuador, hdavidtorob@gmail.com

c. Médico General en funciones Hospitalarias; Hospital Dr. Gustavo Domínguez; Medico; Santo Domingo de los Tsáchilas, Ecuador; monalisatqm@gmail.com

d. Hospital IESS Sur; Urgencia Pediátrica; Médico Cirujano; Quito, Ecuador; dianagalid@ hotmail.com

e. Médico General en funciones Hospitalarias; Hospital Dr. Gustavo Domínguez Zambrano; Médico Cirujano; Santo Domingo de los Tsáchilas, Ecuador; fergigare@ gmail.com

f. Médico Residente de Área de Cirugía Hospital General IESS; Médico Cirujano; Manta, Ecuador; javilifer@ hotmail.com 


\section{Tratamiento de luxación en pacientes con problemas óseos}

Vol. 3, núm. 2., (2019)

Paulina Elizabeth Duran Mora; Hernán David Toro Barrera; Mónica Carolina Calderón Moreira;

Diana Galid San Martín Ajila; Fernanda Gisell García Reyes; Hugo Javier Saltos Giler

\section{RESUMEN}

La estructura esquelética es parte fundamental para el desarrollo y equilibrio del cuerpo humano y a su vez ayuda como sostén de los órganos que lo conforman. Los huesos, como parte fundamental de la estructura ósea, están en un constante crecimiento y regeneración gracias a las células como los osteoblastos, los osteocitos los osteoclastos. Esta regeneración sucede hasta los 35 años de edad pero después de los 40 años empieza un proceso degenerativo donde se hace más vulnerable el sistema óseo. Con esto aparecen los problemas óseos donde los más frecuentes son la artrosis y la osteoporosis. La primera que es la partida de los cartílagos de las articulaciones y la segunda es la pérdida de masa ósea de los huesos. Estos problemas óseos hacen más propenso la aparición de luxaciones. Por lo cual, esta investigación tiene como objetivo general analizar el tratamiento de luxación en pacientes con problemas óseos. La metodología empleada se basó en investigaciones de tipo documental y bibliográfica. Los resultados se basaron en el análisis de las características de la artrosis y de la osteoporosis, como también la determinación del tratamiento de luxación. La conclusión de esta investigación arrojó que una de las características más vulnerables en las personas de la tercera edad es el sufrimiento y dolor en las caderas es por ello que el tratamiento de luxación en las caderas para pacientes que sufren artrosis y osteoporosis se basaran si son con reducción cerrada, que es la más común en su tipo, reducción abierta con o sin desbridamiento y reducción abierta y fijación interna, como también existe tratamiento postreduccinal basado en luxaciones con o sin fractura asociada.

Palabras Claves: Hueso; Estructura Ósea; Artrosis; Osteoporosis; Luxación. 


\title{
Tratamiento de luxación en pacientes con problemas óseos
}

Vol. 3, núm. 2., (2019)

Paulina Elizabeth Duran Mora; Hernán David Toro Barrera; Mónica Carolina Calderón Moreira;

Diana Galid San Martín Ajila; Fernanda Gisell García Reyes; Hugo Javier Saltos Giler

\begin{abstract}
The skeletal structure is a fundamental part for the development and balance of the human body and in turn helps support the organs that comprise it. Bones, as a fundamental part of the bone structure, are constantly growing and regenerating thanks to cells such as osteoblasts, osteocytes and osteoclasts. This regeneration happens until 35 years of age but after 40 years a degenerative process begins where the bone system becomes more vulnerable. With this appear the bone problems where the most frequent are osteoarthritis and osteoporosis. The first that is the departure of the cartilage of the joints and the second is the loss of bone mass of the bones. These bone problems make the appearance of dislocations more prone. Therefore, this research has as a general objective to analyze the treatment of dislocation in patients with bone problems. The methodology used was based on documentary and bibliographic research. The results were based on the analysis of the characteristics of osteoarthritis and osteoporosis, as well as the determination of the treatment of dislocation. The conclusion of this investigation showed that one of the most vulnerable characteristics in the elderly is the suffering and pain in the hips, that is why the treatment of dislocation in the hips for patients suffering from osteoarthritis and osteoporosis will be based on whether they are with closed reduction, which is the most common in its type, open reduction with or without debridement and open reduction and internal fixation, as there is also postreduccinal treatment based on dislocations with or without associated fracture.
\end{abstract}

Key Words: Bone; Bone Structure; Osteoarthritis; Osteoporosis; Dislocation. 


\section{Tratamiento de luxación en pacientes con problemas óseos}

Vol. 3, núm. 2., (2019)

Paulina Elizabeth Duran Mora; Hernán David Toro Barrera; Mónica Carolina Calderón Moreira;

Diana Galid San Martín Ajila; Fernanda Gisell García Reyes; Hugo Javier Saltos Giler

\section{Introducción.}

El crecimiento sano es una de las etapas más importantes en el desarrollo humano ya que dependerá el desarrollo de las actividades en la adultez. La estructura ósea no solo produce el sostén de todos los órganos del cuerpo humano, sino que tiene un funcionamiento que le permite regenerarse ante cualquier adversidad o problema. Recientemente se ha descubierto que el hueso es un tejido donde a nivel molecular existe remodelación continua que restaura la arquitectura para conservar su fortaleza y mantener una permanente comunicación con otros tejidos y sistemas participantes en el equilibrio metabólico (Zárate, Basurto, \& Saucedo, 2010).

Para que el hueso tenga un crecimiento, remodelación y reparación constante durante intervalos de tiempo y dependiendo del caso es importante conocer como el mecanismo que permite esta funcionabilidad. Estas funciones se realizan principalmente por tres tipos de células: osteoblastos (activos en la formación de la matriz ósea), osteocitos (participan en las funciones metabólicas) y osteoclastos (funcionan en la resorción ósea)(Dotor \& Domínguez Hernández, 2002).

Es por ello que una de las etapas más cruciales dentro del crecimiento es el desarrollo de la estructura ósea. El desarrollo del hueso depende de su actividad metabólica, incluyendo la formación y la resorción ósea, por lo cual esta información puede ayudar a prevenir una fragilidad ósea aumentada y el riesgo de fracturas traumáticas de los huesos(Gracia-Marco, y otros, 2009).

"La formación de hueso resulta de un proceso complejo que incluye proliferación de células de la mesénquima, maduración a osteoblastos, formación de matriz orgánica (compuesta 


\section{Tratamiento de luxación en pacientes con problemas óseos}

Vol. 3, núm. 2., (2019)

Paulina Elizabeth Duran Mora; Hernán David Toro Barrera; Mónica Carolina Calderón Moreira; Diana Galid San Martín Ajila; Fernanda Gisell García Reyes; Hugo Javier Saltos Giler

principalmente de fibras de colágena, proteoglicanos, factores de crecimiento, proteínas no colágenas como la osteocalcina, osteopontina, sialoproteínas etc.) y finalmente la mineralización de la matriz caracterizada por depósitos de cristales de hidroxiapatita".(Dotor \& Domínguez Hernández, 2002, pág. 225)

Ahora poder analizar la estructura del hueso desde sus propiedades es vital para el desarrollo de los mecanismos de funcionamiento. Es una estructura material rígida y, sin embargo, con gran plasticidad morfológica, además, es un mecanismo que evita su rápido envejecimiento con la muerte de los osteocitos y microlesiones por fatiga(FORRIOL, 2005). Por lo que, la remodelación asegura, por una parte, la fortaleza, flexibilidad y ductilidad del esqueleto y, por otra parte, interviene en los procesos metabólico-hormonales(Zárate, Basurto, \& Saucedo, 2010).

Es por ello que las fuerzas y cargas ejercidas durante la vida pueden generar problemas que pueden afectar el crecimiento y desarrollo de la estructura ósea. Si las cargas mecánicas aplicadas no se ejecutan con prevención pueden ocurrir daños.

"El microdaño es generado por las cargas cíclicas diarias a las cuales se someten los huesos; dicho microdaño es remodelado por sistemas naturales biológicos. Estos sistemas naturales son procesos de adaptación mediante los cuales el tejido óseo altera su masa y estructura para adaptarse al entorno mecánico al que se encuentra sometido. Aunque esta teoría es mundialmente aceptada, aún no se comprenden los mecanismos mediante los cuales se comienza dicho proceso adaptativo”. (Vera Velandia \& Tovar Pérez, 2008, pág. 73) 


\section{Tratamiento de luxación en pacientes con problemas óseos}

Vol. 3, núm. 2., (2019)

Paulina Elizabeth Duran Mora; Hernán David Toro Barrera; Mónica Carolina Calderón Moreira;

Diana Galid San Martín Ajila; Fernanda Gisell García Reyes; Hugo Javier Saltos Giler

También puede suceder, dentro de la estructura esquelética, dolores articulares que desembocaran en los diferentes problemas óseos que pueda presentar un individuo. El dolor articular es considerado frecuentemente como un dolor puramente nociceptivo y un modelo para la evaluación de analgésicos dirigidos al dolor nociceptivo como los AINEs y los opioides(Perrot, 2016).

Puede producir dolor cuando en un momento determinado el proceso de remodelación produce resorción. La resorción puede predominar sobre la formación y producir enfermedades involutivas, como la osteoporosis(FORRIOL, 2005). Otros de las enfermedades que puede producir dolor dentro de estructura ósea es la artrosis. El dolor por artrosis ha demostrado la activación cerebral el cual se asocia con sensibilización central los cuales son producidos por estímulos que habitualmente no inducen dolor (alodinia) con activación de grandes áreas y una duración mayor del dolor (Perrot, 2016).

Es decir, que indudablemente se producen problemas óseos en los individuos y entre ellos se destaca tanto la artrosis y la osteoporosis de aquí es necesario poder definirlas para poder comprenderlas. La artrosis es una enfermedad osteoarticular crónica y prevalente producida por un proceso degenerativo del cartílago articular y los cambios reactivos en las estructuras óseas periarticulares adyacentes (López, 2006).La osteoporosis es una enfermedad de los huesos, que se caracteriza por disminución de la masa ósea condicionando su fragilidad y función inadecuada de soporte mecánico, también proviene del Osteo = hueso, porosis = poroso: huesos porosos, o con características de cavidades “frágiles”(Padrón, González, de León, Fiallo, \& Kim, 2006). 


\section{Tratamiento de luxación en pacientes con problemas óseos}

Vol. 3, núm. 2., (2019)

Paulina Elizabeth Duran Mora; Hernán David Toro Barrera; Mónica Carolina Calderón Moreira; Diana Galid San Martín Ajila; Fernanda Gisell García Reyes; Hugo Javier Saltos Giler

Ahora, si el individuo con una patología de problemas óseos sufre alguna lesión articular es importante conocer sus causas, síntomas y diagnósticos para poder determinar el tratamiento para poder garantizar una mejor calidad de vida. Cuando estas lesiones articulares se produce la salida de un hueso de su cavidad natural entonces se presenta una luxación(INSHT, 2019).

Por lo tanto, el objetivo general de esta investigación es analizar el tratamiento de luxación en pacientes con problemas óseos. La metodología empleada se basó en una estructura de tipo documental y bibliográfica.

\section{Método.}

Dos de los principales problemas de la estructura ósea es la artrosis y la osteoporosis las cuales son más propensas de sufrir en la edad avanzada y por lo cual son más frecuentes de padecer luxaciones. Estas enfermedades pueden alterar el equilibrio mecánico y por ende disminuir sus propiedades, es entonces la cadera un punto importante para poder determinar un tratamiento de luxación en esa zona. Es por ello, que la metodología empleada para esta investigación es basada en aspectos documentales y bibliográficos tomando en consideración los medios como textos, documentos y artículos científicos publicados disponibles en la web.

\section{Resultados}

Características de la artrosis

Una de las características más comunes en las personas de la tercera edad es la incapacidad para trabajar por padecimiento de artrosis la cual la convierte en un problema de salud pública. La artrosis es la artropatía más frecuente en la población, hasta el punto de que 


\section{Tratamiento de luxación en pacientes con problemas óseos}

Vol. 3, núm. 2., (2019)

Paulina Elizabeth Duran Mora; Hernán David Toro Barrera; Mónica Carolina Calderón Moreira;

Diana Galid San Martín Ajila; Fernanda Gisell García Reyes; Hugo Javier Saltos Giler

más del $70 \%$ de los mayores de 50 años tiene signos radiológicos de artrosis en alguna localización, un dato que debe ser considerado en poblaciones que envejecen(Carmona Ortells, 2010).

Uno de los conceptos más sencillo de esta enfermedad es que afecta las articulaciones de los huesos. Está localizada en las manos, las rodillas, la cadera o la columna vertebral, lo cual provoca dolor, inflamación e impide que se puedan realizar con normalidad algunos movimientos tan cotidianos como cerrar la mano, subir escaleras o caminar(Mamani Conde, 2018).El patrón distributivo varía por sexos; la artrosis de rodillas y manos predomina en las mujeres, habiendo un ligero predominio masculino en la artrosis de cadera(Morgado, Pérez, Moguel, Pérez-Bustamante, \& Torres, 2005). En la Tabla 1 se detalla los criterios clínicos dependiendo de la distribución de la artrosis. 


\title{
Tratamiento de luxación en pacientes con problemas óseos
}

Vol. 3, núm. 2., (2019)

Paulina Elizabeth Duran Mora; Hernán David Toro Barrera; Mónica Carolina Calderón Moreira; Diana Galid San Martín Ajila; Fernanda Gisell García Reyes; Hugo Javier Saltos Giler

Tabla 1. Criterios clínicos dependiendo de la distribución de la artrosis, según el American

\section{Collegeof Rheumatology}

\author{
Artrosis de rodilla ${ }^{14}$ \\ Dolor de rodillas acompañado de, al menos, 4 de estos otros 6 criterios: \\ - Edad superior a 50 affos \\ - Rigidez matutina de menos de 30 minutos \\ - Crepitación ósea a los movimientos activos \\ - Dolor a la presión sobre los márgenes óseos de la articulación \\ - Hipertrofia articular de consistencia dura \\ - Ausencia de síntomas evidentes de inflamación \\ Artrosis de $\operatorname{mano}^{13}$ \\ Dolor, molestias o rigidez de las articulaciones de las manos, acompafiado de \\ - Hipertrofia articular de consistencia dura de 2 o más de 10 articulaciones seleccionadas (interfalángicas distales y \\ proximales de los dedos $2 .^{\circ}$ y $3 .^{\circ}$ y las trapeciometacarpianas de ambas manos) \\ - Signos de tumefacción presentes en menos de 3 articulaciones metacarpofalángicas \\ - Uno de estos otros 2 criterios \\ - Hipertrofia articular de consistencia dura de 2 o más articulaciones interfalángicas distales (cualquier dedo) \\ - Deformidad de 20 más de las 10 articulaciones seleccionadas (interfalángicas distales y proximales de los \\ dedos $2 .^{\circ}$ y $3 .^{\circ} \mathrm{y}$ las trapeciometacarplanas de ambas manos) \\ Artrosis de cadera ${ }^{12}$ (criterios clínicos y radiográficos) \\ Coxalgia presente la mayor parte de los dias del mes previo, más 2 de los 3 criterios siguientes: \\ -VSG $\leq 20 \mathrm{~mm} / \mathrm{h}$ \\ - Osteofitos femorales o acetabulares en radiografía \\ - Pinzamiento de la interlínea en radiografía
}

Fuente: (Carmona Ortells, 2010)

También tiene un concepto operativo que radica en la pérdida del cartílago presente en las articulaciones. Una condición de las articulaciones sinoviales caracterizada por la pérdida de cartílago (condropatía) y evidencia de una respuesta ósea periarticular», a todas luces, imposible de utilizar desde el punto de vista epidemiológico(Carmona Ortells, 2010).

Por lo cual radica la importancia del cartílago como factor determinante para el padecimiento de artrosis. El cartílago es el tejido encargado de recubrir los extremos de estos 


\section{Tratamiento de luxación en pacientes con problemas óseos}

Vol. 3, núm. 2., (2019)

Paulina Elizabeth Duran Mora; Hernán David Toro Barrera; Mónica Carolina Calderón Moreira;

Diana Galid San Martín Ajila; Fernanda Gisell García Reyes; Hugo Javier Saltos Giler

huesos y es indispensable para el buen funcionamiento de la articulación puesto que actúa como un amortiguador(Mamani Conde, 2018). Esta se puede observar en la Figura 1.

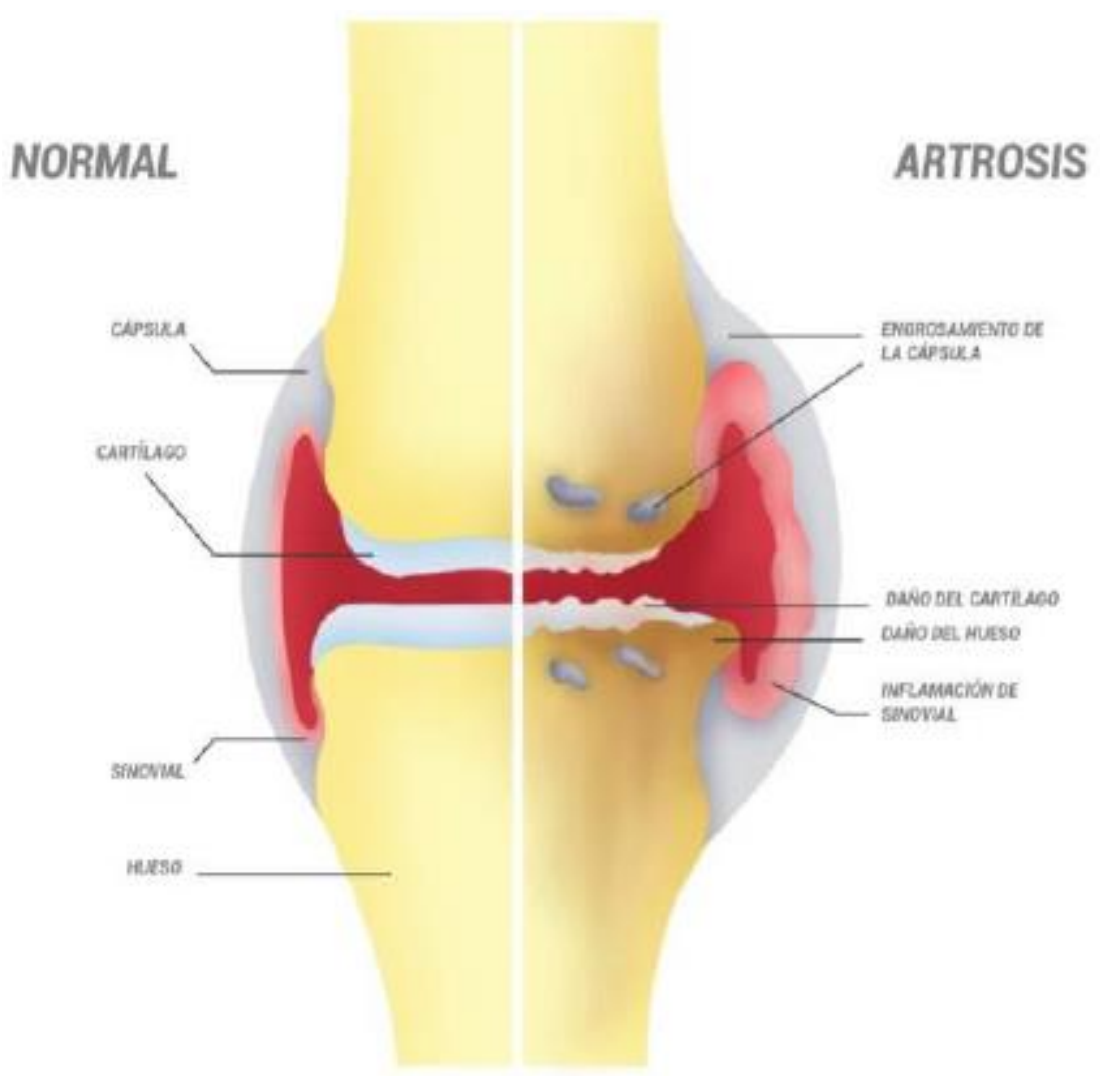

Figura 1. Unión de la articulación de la rodilla, comparación de una sana y otra con artrosis

Fuente: (Mamani Conde, 2018).

De aquí se puede definir más claramente el problema de artrosis en las personas de avanzada edad. La artrosis provoca el deterioro del cartílago articular haciendo que los huesos se vayan desgastando y aparezca el dolor, ya que a medida que el cartílago va desapareciendo, el hueso reacciona y crece por los lados (osteofitos) produciendo la deformación de la articulación(Mamani Conde, 2018). También se puede definir como una enfermedad del aparato 


\section{Tratamiento de luxación en pacientes con problemas óseos}

Vol. 3, núm. 2., (2019)

Paulina Elizabeth Duran Mora; Hernán David Toro Barrera; Mónica Carolina Calderón Moreira; Diana Galid San Martín Ajila; Fernanda Gisell García Reyes; Hugo Javier Saltos Giler

locomotor caracterizada por la degeneración y pérdida del cartílago articular, junto a la proliferación osteocartilaginosa subcondral y de los márgenes articulares(Morgado, Pérez, Moguel, Pérez-Bustamante, \& Torres, 2005).

Existen manifestaciones clínicas de esta enfermedad las cuales se caracterizan por el dolor. Las causas del dolor son múltiples, debiéndose explicar tanto por la afectación articular propiamente dicha (afectación cartílago articular, hueso subcondral, sinovial, meniscos, etc.), como por la afectación periarticular (ligamentos, estructuras tendinosas, etc.)(López, 2006).El síntoma más importante es el dolor, de características mecánicas, apareciendo con la deambulación y mejorando con el reposo, si bien en las formas severas el dolor puede aparecer incluso en reposo, pudiendo despertar al paciente durante la noche(Morgado, Pérez, Moguel, Pérez-Bustamante, \& Torres, 2005).

Existen factores que aumentan la incidencia y progreso de la artrosis, los cuales se pueden manifestar en generales intrínsecos y extrínsecos.

"Los generales son edad, sexo y genética, los intrínsecos son la falta de ensamblado (como las displasias) y la laxitud articular que causa desgaste y soporte del peso anormales y por ello aceleran la degeneración articular, y los extrínsecos que son el índice de masa corporal en aumento, gran cantidad de ciertos ejercicios y el trabajo manual pesado”. (Aresti, 2016, pág. 1)

La artrosis produce una reducción en la movilidad ya que la intensidad del dolor va en aumento según la severidad del daño. Esta limitación es producida por las reacciones osteofíticas periarticulares y las contracturas musculares secundarias, que si evolucionan condicionan una 


\section{Tratamiento de luxación en pacientes con problemas óseos}

Vol. 3, núm. 2., (2019)

Paulina Elizabeth Duran Mora; Hernán David Toro Barrera; Mónica Carolina Calderón Moreira;

Diana Galid San Martín Ajila; Fernanda Gisell García Reyes; Hugo Javier Saltos Giler

incapacidad importante como es el caso de la articulación de la cadera y rodilla (contractura en flexión antiálgica)(López, 2006).La rigidez articular matutina, que puede durar hasta 30 minutos tras el inicio de la movilización articular, los crujidos articulares, la inestabilidad y la contractura muscular, todo ello acompañado de una ausencia de sintomatología sistémica(Morgado, Pérez, Moguel, Pérez-Bustamante, \& Torres, 2005). Dependiendo de los signos se puede llegar a un diagnostico como se muestra en la Tabla 2.

Ahora es necesario poder determinar un diagnóstico de la artrosis. El diagnóstico se basa en la conjunción de manifestaciones clínicas, hallazgos exploratorios y signos radiológicos, junto a valores de laboratorio dentro de la normalidad (Morgado, Pérez, Moguel, Pérez-Bustamante, \& Torres, 2005).

Tabla 2. Signos y síntomas de la artrosis y posible diagnostico según el caso

\begin{tabular}{lll}
\hline SíNTOMA & Posible DIAGNÓSTICO \\
\hline Dolor persistente y progresivo que no varía con la actividad o el tiempo & Tumor maligno o infección \\
\hline Dolor noctumo & Tumor maligno o infección \\
\hline Dolor de reposo & Tumor maligno o infección \\
\hline Rigidez matinal que dura $>2$ horas & Artritis inflamatoria \\
\hline Antecedentes de cáncer & Metástasis \\
\hline Antecedentes de enfermedad del tejido conectivo & Artritis inflamatoria \\
\hline
\end{tabular}

Fuente: (Aresti, 2016)

La radiología es el método más sencillo y económico de los métodos o técnicas diagnósticas para determinar el padecimiento de artrosis. Es una prueba para realizar un diagnóstico correcto e incluso para realizar el seguimiento de la progresión en el tiempo donde es 


\section{Tratamiento de luxación en pacientes con problemas óseos}

Vol. 3, núm. 2., (2019)

Paulina Elizabeth Duran Mora; Hernán David Toro Barrera; Mónica Carolina Calderón Moreira; Diana Galid San Martín Ajila; Fernanda Gisell García Reyes; Hugo Javier Saltos Giler

básico realizar el estudio comparativo de las articulaciones contralaterales para esta enfermedad (López, 2006). Esta se puede observar en la Figura 2 donde se muestra una radiografía anteroposterior del hombro derecho que demuestra osteofito inferior de la cabeza humeral mayor a $7 \mathrm{~mm}$.

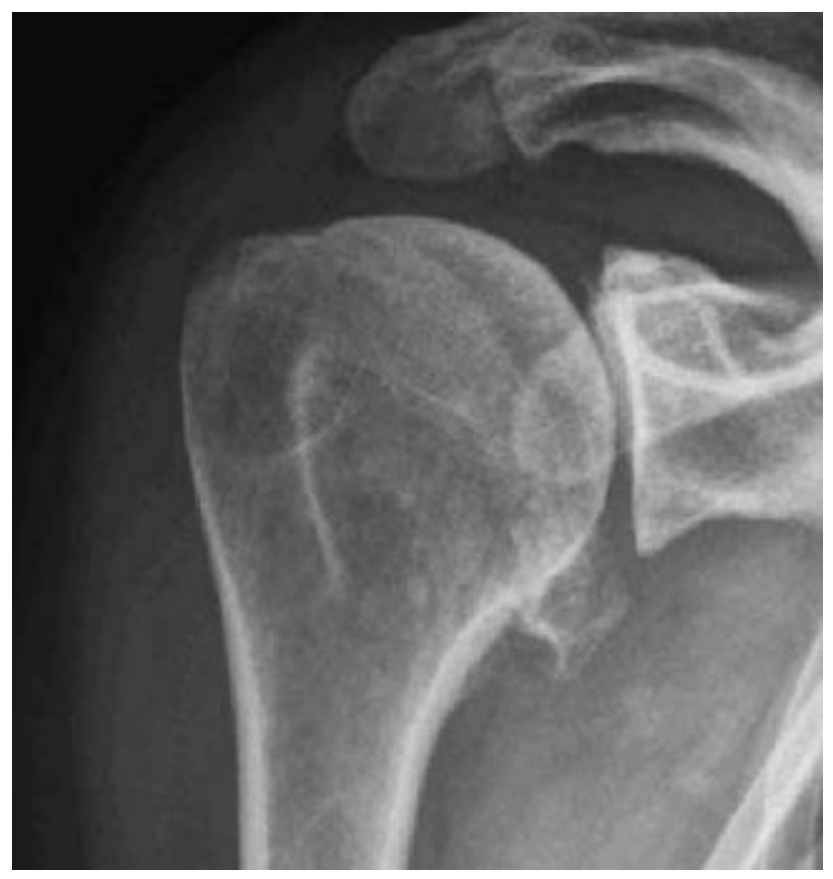

Figura 2. Radiografía anteroposterior del hombro derecho que demuestra osteofito inferior de la cabeza humeral mayor a $7 \mathrm{~mm}$

Fuente: (Gutiérrez \& Ekdahl, 2014)

Aunado a este tipo de examen radiológico es recomendable tomar muestras de laboratorio.

"Se dispone de un examen de analítica general (hemograma, velocidad de sedimentación, bioquímica general, etc.), donde se puede estudiar el líquido sinovial, de características viscoso y con pocas células en su mayoría mononucleares, para descartar otras enfermedades 


\section{Tratamiento de luxación en pacientes con problemas óseos}

Vol. 3, núm. 2., (2019)

Paulina Elizabeth Duran Mora; Hernán David Toro Barrera; Mónica Carolina Calderón Moreira;

Diana Galid San Martín Ajila; Fernanda Gisell García Reyes; Hugo Javier Saltos Giler

coexistentes ya que la presencia de un derrame pequeño o moderado es común”.(López, 2006, pág. 3)

Existen otros tipos de pruebas las cuales van a depender de la severidad de daño que tenga el paciente. Estas pruebas son la tomografía axial computarizada (TAC), resonancia magnética nuclear (RMN) y artroscopia. La primera es complemento de la radiografía (ver Figura 3), la segunda es una técnica que favorece el estudio óseo y de partes blandas y el tercero es una técnica invasiva que permite visualizar las diferentes afecciones articulares previas a los cambios radiológicos (ver Figura 4)(López, 2006).

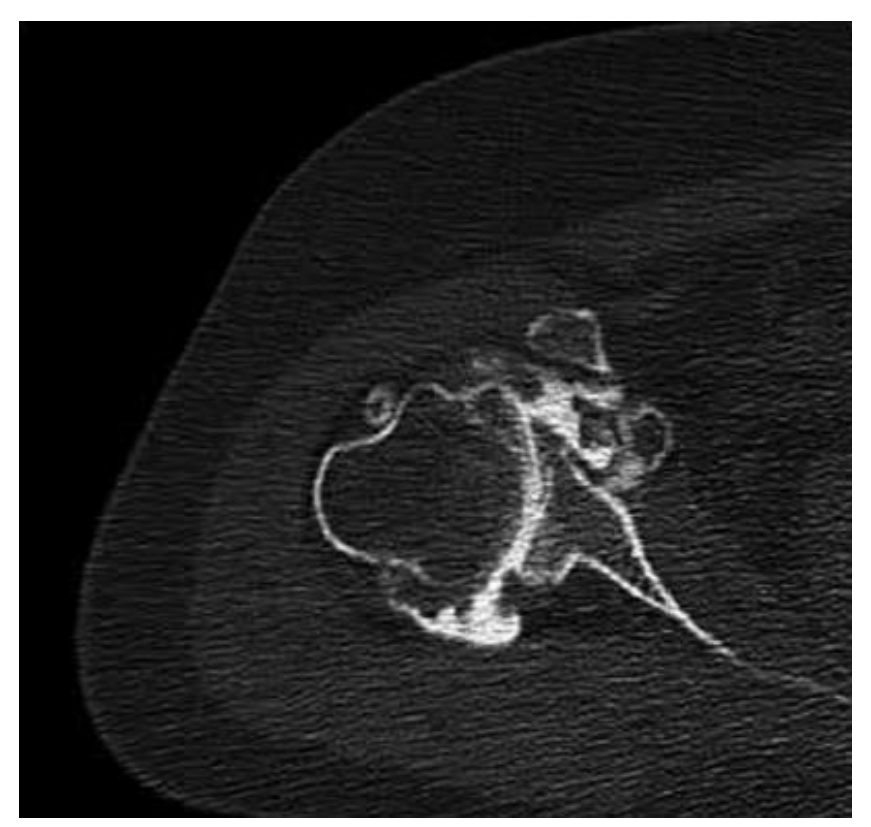

Figura 3. Imagen de TAC con contraste del hombro derecho, que muestra el corte axial disminución del espacio articular glenohumeral y osteofitos

Fuente: (Gutiérrez \& Ekdahl, 2014) 


\section{Tratamiento de luxación en pacientes con problemas óseos}

Vol. 3, núm. 2., (2019)

Paulina Elizabeth Duran Mora; Hernán David Toro Barrera; Mónica Carolina Calderón Moreira; Diana Galid San Martín Ajila; Fernanda Gisell García Reyes; Hugo Javier Saltos Giler

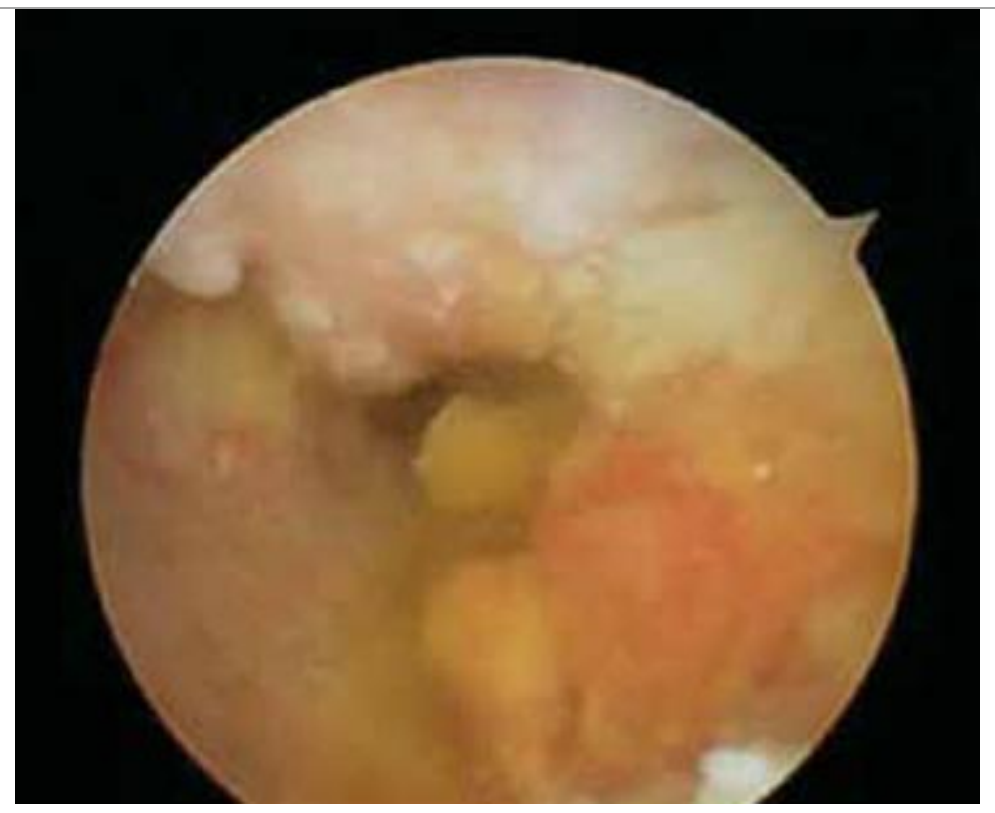

Figura 4. Visión artroscópica de articulación gleno-humeral que muestra sinovitis y denudación de cartílago articular

Fuente: (Gutiérrez \& Ekdahl, 2014)

Como artrosis es más frecuente en rodilla y cadera entonces se plantean unos métodos diagnósticos propuestos por el Colegio Americano de Reumatología los cuales presentan una excelente respuesta para pacientes que sufren de esta enfermedad. Estos se muestran en la Figura 5 y Figura 6, respectivamente. 


\section{Tratamiento de luxación en pacientes con problemas óseos}

Vol. 3, núm. 2., (2019)

Paulina Elizabeth Duran Mora; Hernán David Toro Barrera; Mónica Carolina Calderón Moreira;

Diana Galid San Martín Ajila; Fernanda Gisell García Reyes; Hugo Javier Saltos Giler

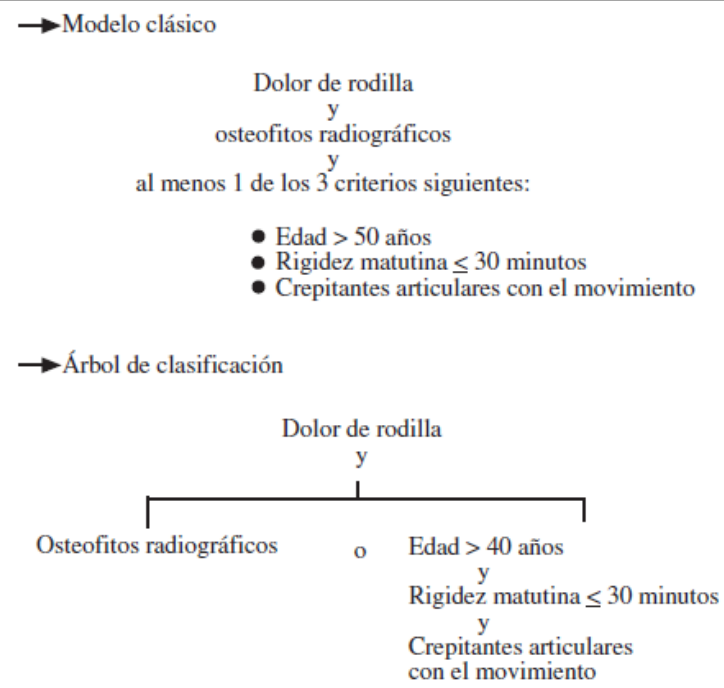

Figura 5. Criterios diagnósticos para la artrosis de rodilla

Fuente: (Morgado, Pérez, Moguel, Pérez-Bustamante, \& Torres, 2005)

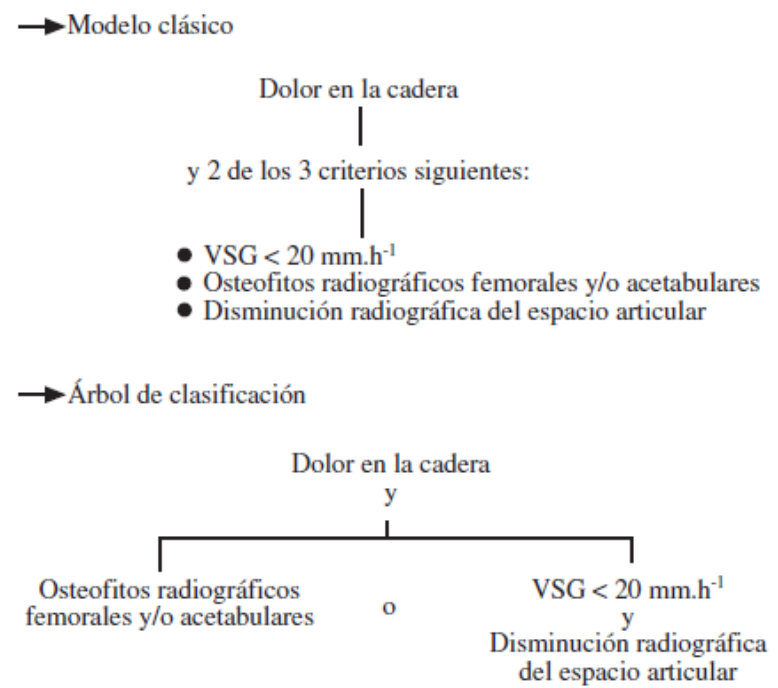

Figura 6. Criterios diagnóstico para la artrosis de cadera

Fuente: (Morgado, Pérez, Moguel, Pérez-Bustamante, \& Torres, 2005) 


\section{Tratamiento de luxación en pacientes con problemas óseos}

Vol. 3, núm. 2., (2019)

Paulina Elizabeth Duran Mora; Hernán David Toro Barrera; Mónica Carolina Calderón Moreira;

Diana Galid San Martín Ajila; Fernanda Gisell García Reyes; Hugo Javier Saltos Giler

\section{Características de la osteoporosis}

Otra de las enfermedades más comunes en la estructura ósea es la osteoporosis la cual produce una disminución de la calidad de vida de las personas en avanzada edad. Es una enfermedad esquelética sistémica, caracterizada por baja masa ósea y deterioro en la micro arquitectura del tejido óseo, que origina fragilidad ósea aumentada con el consecuente aumento en el riesgo de fractura(Gabrielli \& Rodríguez, 2004).Existe una definición bien exacta de esta enfermedad ofrecida por el National Institute of Health.

"La osteoporosis se define como una enfermedad generalizada del sistema esquelético caracterizada por la pérdida de masa ósea y por el deterioro de la microestructura del tejido óseo, que compromete la resistencia ósea y que condiciona como consecuencia una mayor fragilidad ósea y una mayor susceptibilidad a las fracturas”. (Hermoso de Mendoza, 2003, pág. 30)

Estas características de adelgazamiento del tejido óseo y disminución de la resistencia de los huesos se pueden observar en la Figura 7. La osteoporosis no tiene un comienzo bien definido y, hasta hace poco, el primer signo visible de la enfermedad acostumbraba a ser una fractura de la cadera, la muñeca o de los cuerpos vertebrales que originaban dolor o deformidad(Padrón, González, de León, Fiallo, \& Kim, 2006). 


\section{Tratamiento de luxación en pacientes con problemas óseos}

Vol. 3, núm. 2., (2019)

Paulina Elizabeth Duran Mora; Hernán David Toro Barrera; Mónica Carolina Calderón Moreira;

Diana Galid San Martín Ajila; Fernanda Gisell García Reyes; Hugo Javier Saltos Giler

(A)

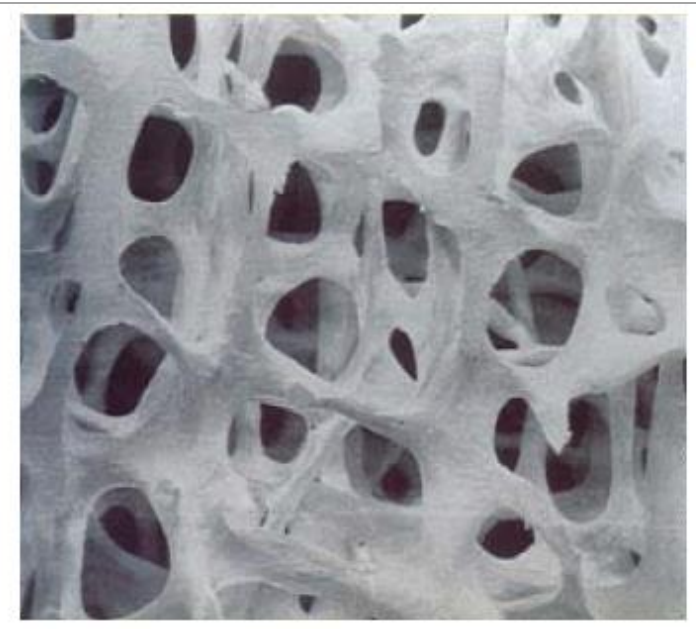

(B)

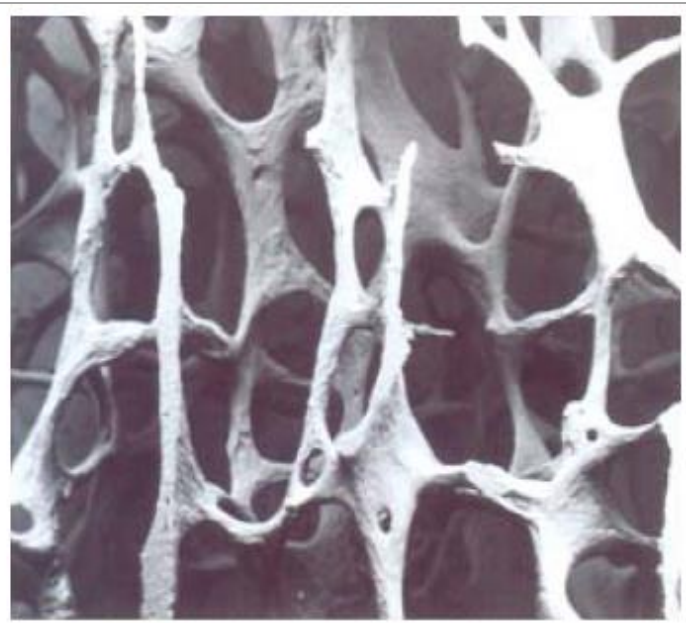

Figura 7. Hueso trabecular. (A) Normal y (B) osteoporótico

Fuente: (Martínez, Molina, Consuegra, Vázquez, \& Fernández, 2006)

Es importante definir las edades que permitan un desarrollo óseo ya que alguna alteración permite un cambio en la salud en el individuo. Los huesos están en un constante estado de renovación, lo cual es producido por dos tipos de células, osteoblastos y osteoclastos, donde el primero forma el hueso y el segundo lo descomponen, en un proceso llamado reabsorción(Padrón, González, de León, Fiallo, \& Kim, 2006). Después de 40 años, se rompe el equilibrio producción más reabsorción que la formación ósea.

"Cuando la masa ósea del adulto alcanza su valor máximo aproximadamente a los 35 años, la tasa de síntesis y de reabsorción ósea es equivalente. Este equilibrio normal entre la síntesis y reabsorción ósea mantiene constante la masa esquelética. A partir de los 40 años se observa una lenta reducción de la densidad de masa ósea en ambos sexos (aproximadamente 0,3-0.5\% al año). Se desconocen las razones por las que se produce esta pérdida ósea con la edad, y porque se inicia a una edad más temprana en las mujeres y sigue en curso más acelerado 


\section{Tratamiento de luxación en pacientes con problemas óseos}

Vol. 3, núm. 2., (2019)

Paulina Elizabeth Duran Mora; Hernán David Toro Barrera; Mónica Carolina Calderón Moreira;

Diana Galid San Martín Ajila; Fernanda Gisell García Reyes; Hugo Javier Saltos Giler

incluso antes de la menopausia. Sin embargo, un individuo que no alcance un pico de masa ósea optimo durante la infancia y adolescencia puede padecer osteoporosis sin que se produzca una pedida acelerada de masa ósea”.(Hermoso de Mendoza, 2003, pág. 30)

Ahora, los pacientes que empiezan a sufrir de osteoporosis empiezan a tener síntomas ya cuando el problema a avanzado, es decir que a los primeros momentos no afecta debido a que la disminución de la masa ósea ocurre de manera muy lenta, pero al pasar el tiempo y la edad del individuo los síntomas se recrudecen. En la espalda, el dolor aparece por el aplastamiento de las vértebras. El dolor empieza de manera súbita, se localiza en una zona determinada de la espalda y empeora al estar de pie o al caminar, como también puede aparecer dolor al tacto y, habitualmente, desaparece de forma gradual al cabo de un tiempo(Padrón, González, de León, Fiallo, \& Kim, 2006).

Existen dos tipos de osteoporosis primaria. La osteoporosis tipo I y el tipo II. Donde cada una se diferencia por edad, sexo, zona de pérdida ósea, fractura, entre otros. Esto se puede observar en la Tabla 3.También existe la osteoporosis secundaria la cual aparecen como una manifestación acompañante de otras enfermedades o de su tratamiento (Hermoso de Mendoza, 2003). 


\section{Tratamiento de luxación en pacientes con problemas óseos}

Vol. 3, núm. 2., (2019)

Paulina Elizabeth Duran Mora; Hernán David Toro Barrera; Mónica Carolina Calderón Moreira;

Diana Galid San Martín Ajila; Fernanda Gisell García Reyes; Hugo Javier Saltos Giler

\section{Tabla 3. Diferencias de las osteoporosis tipo I y tipo II}

\begin{tabular}{|c|c|c|}
\hline & TIPO I & TIPO II \\
\hline Edad & $51-75$ & 70 y más \\
\hline Razóm mujer/hombre & $6: 1$ & $2: 1$ \\
\hline Tipo de pérdida ósea & Primariamente trabecular & Trabecular cortical \\
\hline Razón de pérdida ósea & Acelerada/ corta duración. & No acelerada/larga duración. \\
\hline Sitio de fractura & $\begin{array}{l}\text { Vértebras (aplastamiento) } \\
\text { radio distal (Fractura de } \\
\text { Colles) }\end{array}$ & Vértebra (en cuña) y fémur \\
\hline $\begin{array}{l}\text { Valores de laboratorio } \\
\text { Calcio sérico }\end{array}$ & Normal & Normal \\
\hline Fosfatasa sérica & Normal & Normal \\
\hline Fosfatasa alcalina & $\begin{array}{l}\text { Normal (aumenta con la } \\
\text { fractura). }\end{array}$ & $\begin{array}{l}\text { Normal (aumenta con la } \\
\text { fractura). }\end{array}$ \\
\hline Calcio urinario & Aumentado & Normal \\
\hline Función PTH & Disminuida & Aumentada \\
\hline $\begin{array}{l}\text { Metabolismo de } 25(\mathrm{OH}) \mathrm{D} \\
\text { ó } 25 \text { (oh) 2D }\end{array}$ & $\begin{array}{l}\text { Disminuido } \\
\text { secundariamente }\end{array}$ & $\begin{array}{l}\text { Disminuido } \\
\text { secundariamente }\end{array}$ \\
\hline Absorción de calcio & $\begin{array}{l}\text { Disminuida, relacionada con } \\
\text { la menopausia. }\end{array}$ & $\begin{array}{l}\text { Disminuido, relacionado con } \\
\text { el envejecimiento }\end{array}$ \\
\hline
\end{tabular}

Fuente: (Estrada, Gallard, Fernandez, \& Martinez, 1998)

Las causas de la osteoporosis son variadas ya que pueden depender de múltiples enfermedades o hábitos de la vida de la persona, pero una de las principales causas en las mujeres es la menopausia. La menopausia es la principal causa de osteoporosis en las mujeres, debida a disminución de los niveles de estrógenos. La pérdida de estrógenos por la menopausia fisiológica o por la extirpación quirúrgica de los ovarios ocasiona una rápida pérdida de hueso(Padrón, González, de León, Fiallo, \& Kim, 2006). En la Tabla 4 se puede detallar las causas de la osteoporosis secundaria.

Para poder prevenir y poder aplicar un tratamiento es necesario conocer los factores de riesgo de la osteoporosis. Estos se pueden observar en la Tabla 5. 


\section{Tratamiento de luxación en pacientes con problemas óseos}

Vol. 3, núm. 2., (2019)

Paulina Elizabeth Duran Mora; Hernán David Toro Barrera; Mónica Carolina Calderón Moreira; Diana Galid San Martín Ajila; Fernanda Gisell García Reyes; Hugo Javier Saltos Giler

\section{Tabla 4. Causas de la osteoporosis secundaria}

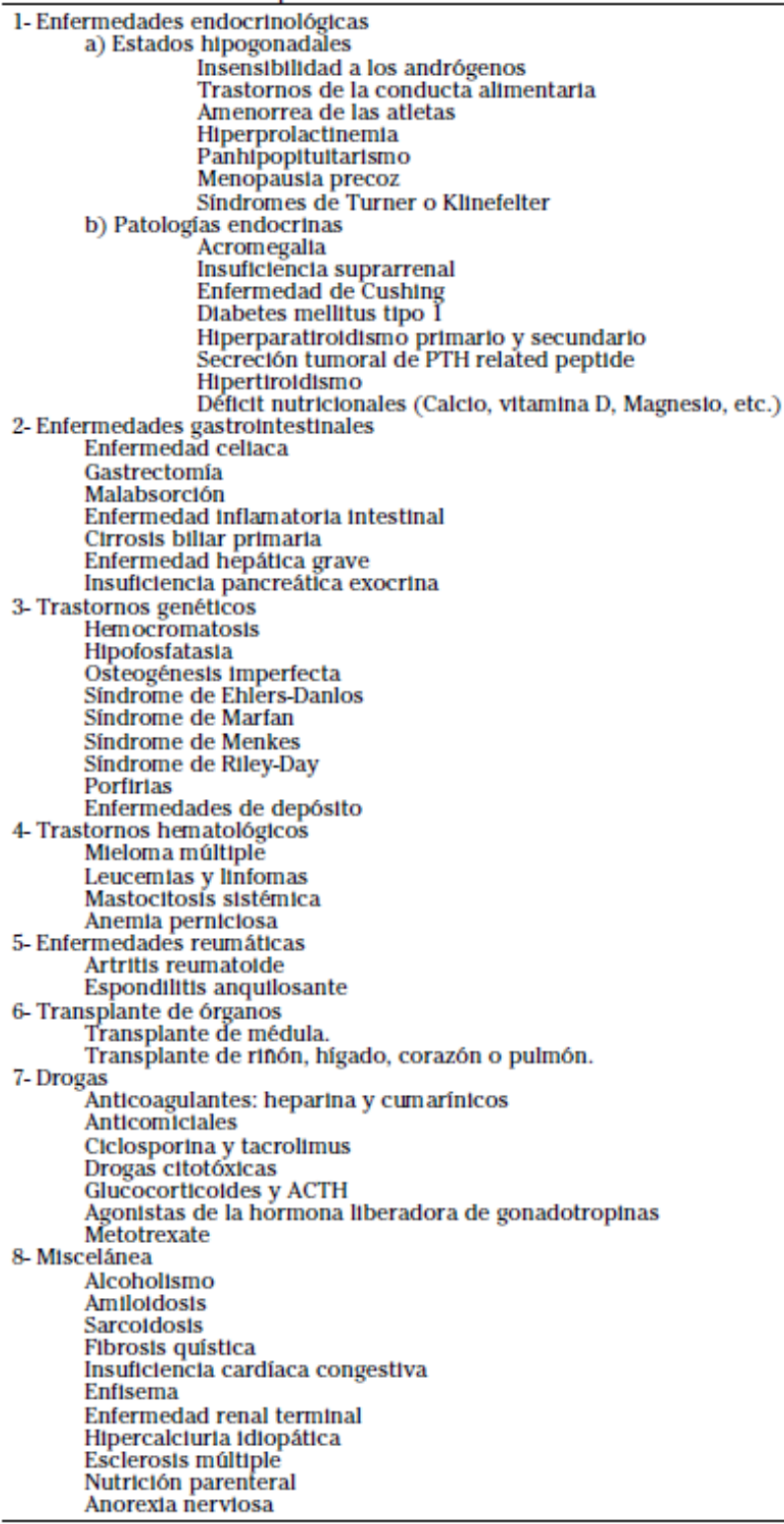

Fuente: (Hermoso de Mendoza, 2003) 
Tratamiento de luxación en pacientes con problemas óseos

Vol. 3, núm. 2., (2019)

Paulina Elizabeth Duran Mora; Hernán David Toro Barrera; Mónica Carolina Calderón Moreira;

Diana Galid San Martín Ajila; Fernanda Gisell García Reyes; Hugo Javier Saltos Giler

\section{Tabla 5. Factores de riesgo de la osteoporosis}

\begin{tabular}{|c|c|}
\hline 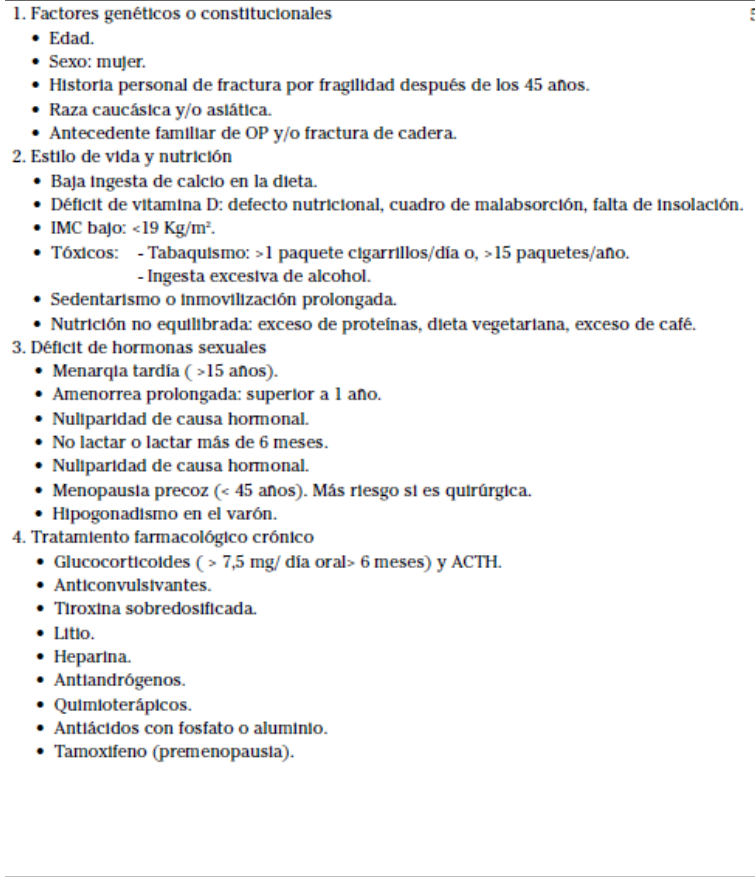 & 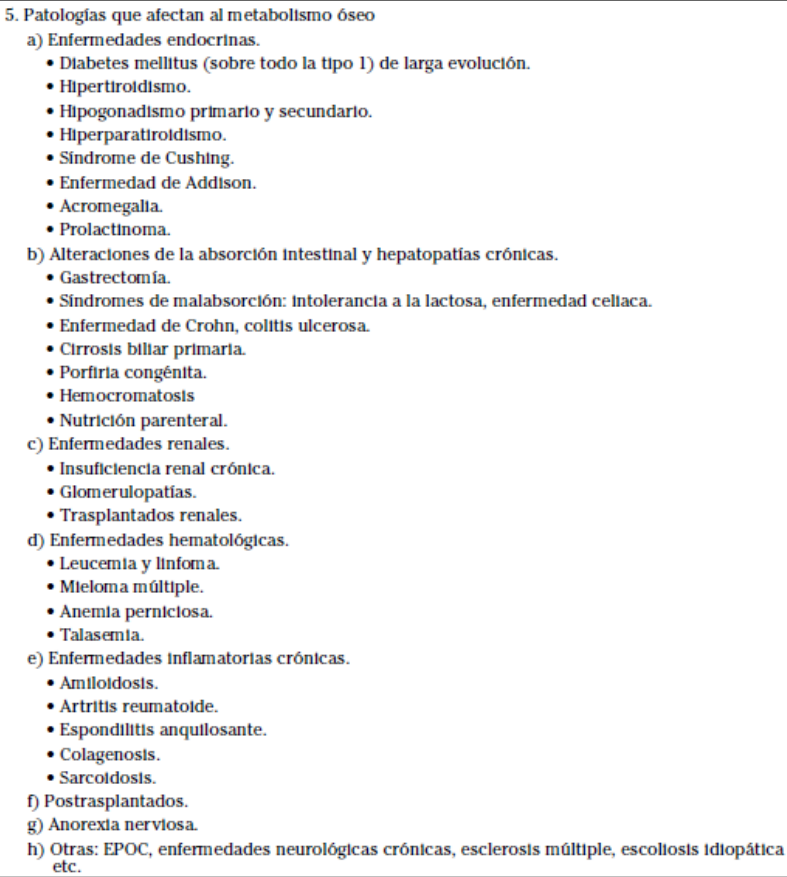 \\
\hline
\end{tabular}

Fuente: (Hermoso de Mendoza, 2003)

Es necesario realizar el diagnóstico de osteoporosis con el fin de liberar sospechas de padecimiento de la enfermedad. La valoración de los factores de riesgo asociados a una masa ósea baja, la existencia de fractura previa o el aumento del riesgo de caídas son datos de gran utilidad en el diagnóstico (Giménez, Zafra, \& González, 2006). En la Figura 8 se detalla el esquema por etapas del diagnóstico de osteoporosis. 


\section{Tratamiento de luxación en pacientes con problemas óseos}

Vol. 3, núm. 2., (2019)

Paulina Elizabeth Duran Mora; Hernán David Toro Barrera; Mónica Carolina Calderón Moreira; Diana Galid San Martín Ajila; Fernanda Gisell García Reyes; Hugo Javier Saltos Giler

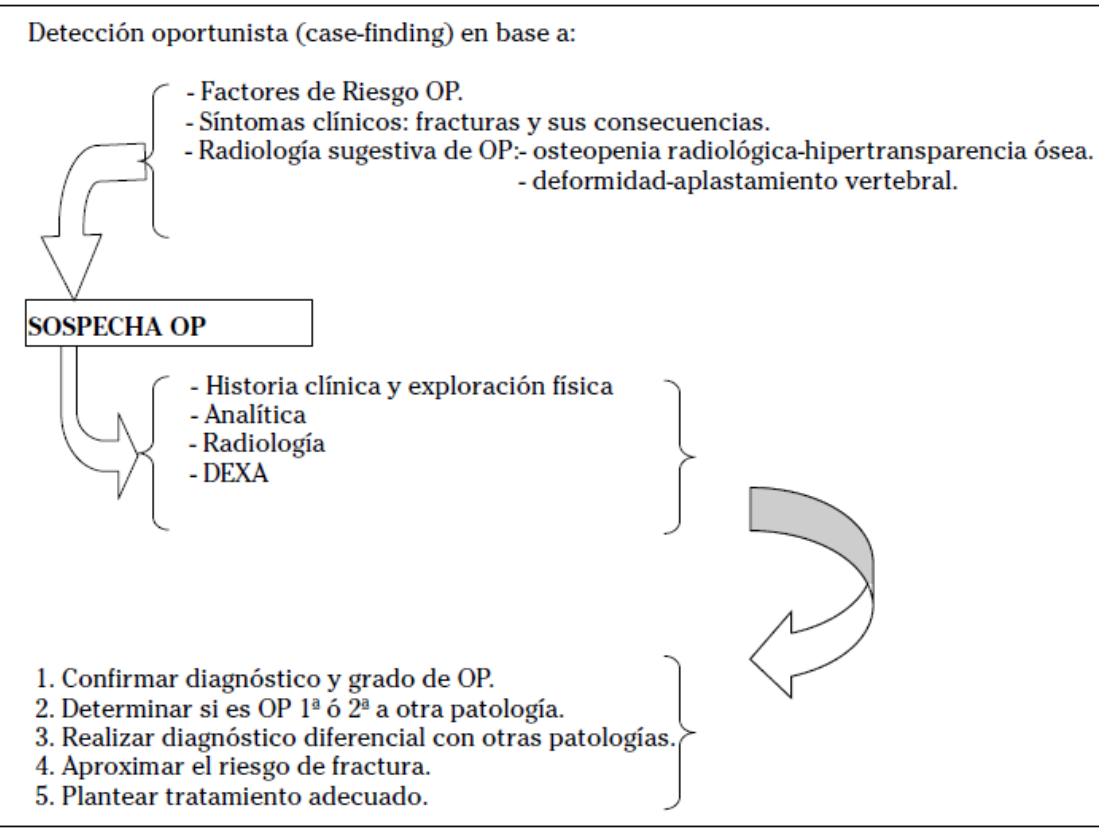

Figura 8. Esquema por etapas del diagnóstico de osteoporosis

Fuente: (Hermoso de Mendoza, 2003)

Para el análisis por diagnóstico de laboratorio ocurre lo siguiente:

"Los niveles séricos de Ca y P, electroforesis de proteínas séricas y VCG son normales en la osteoporosis primaria. La fosfatasa alcalina sérica suele ser normal, aunque en el caso que exista una fractura reciente puede estar aumentada. Los niveles de PTH son normales o bajos en el paciente tipo I y elevados en el tipo II; si desciende la absorción de calcio o si existe una hipercalciuria inadecuadalos indicadores de recambio óseo pueden esta relevados (excreción urinaria de péptidos que contienen hidroxipolina, péptido de piridinio en orina osteocalcinasérica o captación de disfosfonato metileno de tecnecio-99).Otros hallazgos de laboratorios anormales sugieren una osteoporosis secundaria”.(Martínez, Molina, Consuegra, Vázquez, \& Fernández, 2006, pág. 87) 


\section{Tratamiento de luxación en pacientes con problemas óseos}

Vol. 3, núm. 2., (2019)

Paulina Elizabeth Duran Mora; Hernán David Toro Barrera; Mónica Carolina Calderón Moreira;

Diana Galid San Martín Ajila; Fernanda Gisell García Reyes; Hugo Javier Saltos Giler

Otra técnica como la radiografía la cual no ayuda mucho debido a que solo es observable si la pérdida de masa ósea es considerable. Una aparente disminución de densidad ósea en la radiografía no es sinónimo de osteoporosis, sin embargo, debe sospecharse al observar disminución de las trabéculas, disminución del grosor cortical y más aún deformaciones vertebrales o antiguas fracturas (De Reumatología, 2007).

Una técnica diagnóstica importante es la densitometría donde permite la cuantificación de la masa ósea. La cuantificación de la masa ósea es la base diagnóstica de la osteoporosis, siendo la mejor técnica disponible de medición de masa ósea y uno de los predictores más fiables en la estimación del riesgo de fractura(Giménez, Zafra, \& González, 2006). Dentro de estos métodos se encuentra la DXA (Absorciometría dual de rayos X). Es un método que permite medir la densidad ósea (DO) y el contenido mineral (CM) en el cuerpo total y en sitios específicos tanto del esqueleto axial como periférico y específicamente aquellos con mayor riesgo de fractura como columna, cadera y muñeca (De Reumatología, 2007). En la Tabla 6 se muestra las indicaciones actuales de densitometría ósea. 


\section{Tratamiento de luxación en pacientes con problemas óseos}

Vol. 3, núm. 2., (2019)

Paulina Elizabeth Duran Mora; Hernán David Toro Barrera; Mónica Carolina Calderón Moreira;

Diana Galid San Martín Ajila; Fernanda Gisell García Reyes; Hugo Javier Saltos Giler

\section{Tabla 6. Indicaciones actuales de densitometría ósea}

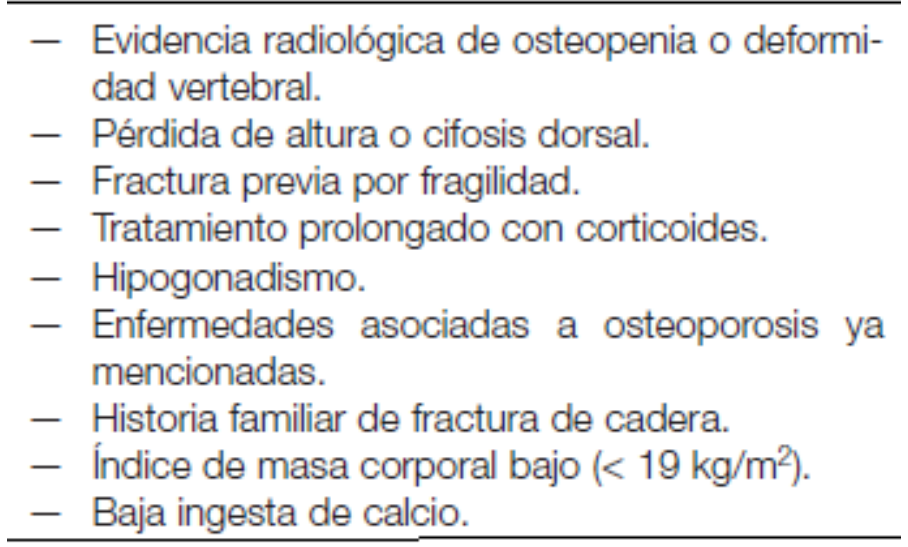

Fuente: (Giménez, Zafra, \& González, 2006)

Luxación en pacientes con problemas óseos

Una de las características de los problemas óseos donde no hay fractura sino un movimiento de las uniones de los huesos son las luxaciones. Esta consiste en el desplazamiento permanente de las superficies articulares de los huesos que componen una articulación, donde es completa si las superficies articulares no tienen contacto e incompleta si hay contacto parcial (Aguilar Caballero \& Galbez García de Aguilar, 1976).Inicialmente se consideraba que las luxaciones puras cursaban con una mejor evolución, pero en reportes recientes se han encontrado resultados insatisfactorios hasta en 50\% de los casos(Fernández Palomo, 2001).

Existen causas por las cuales pueden aparecer luxaciones y se presentan por varios factores. Se presenta con más frecuencia en personas jóvenes y son de característica congénita, espontánea y traumática o por violencia mecánica, donde son mucho menos frecuentes que las fracturas, pero se presentan en las articulaciones de mayor movilidad como hombro cadera y 


\section{Tratamiento de luxación en pacientes con problemas óseos}

Vol. 3, núm. 2., (2019)

Paulina Elizabeth Duran Mora; Hernán David Toro Barrera; Mónica Carolina Calderón Moreira; Diana Galid San Martín Ajila; Fernanda Gisell García Reyes; Hugo Javier Saltos Giler

codo (Aguilar Caballero \& Galbez García de Aguilar, 1976). Pueden producirse con lesiones asociadas tales como distensión de la cápsula articular, ruptura de la cápsula, elongaciones ligamentosas, ruptura total o parcial de los ligamentos y fracturas articulares(Miralles, 2010).

“Los síntomas son el dolor, la hinchazón y el amoratamiento, En la luxación no hay movilidad pudiendo presentar deformidad en la parte afectada. El entumecimiento o la parálisis por debajo de la localización de la luxación debe alertarnos sobre la existencia de sufrimiento vascular o nervioso”. (INSHT, 2019, pág. 7)

Los problemas óseos en los individuos producen el debilitamiento de las articulaciones y pérdida de masa ósea en los huesos manifestados a través a de la artrosis y osteoporosis. Esto sucede más frecuentemente en la cadera a medida que avanza la edad en el paciente. Es por ello que es necesario determinar las características de las luxaciones en caderas para personas con problemas óseos.

"La cadera es una articulación esférica que permite movimientos de flexo-extensión, abducción-aducción, rotacionales y de circunducción. La cabeza femoral representa 2/3 de una esfera y está recubierta de cartílago. El acetábulo del hueso coxal tiene una superficie articular en forma de herradura, que se dispone en arco alrededor de la fosa acetabular”.(Payo Rodríguez, Ibarzabal Gil, \& Rodríguez Merchán, 2006, pág. 168)

Cuando hay un impacto repentino y de gran magnitud produce que la cadera sufra un trauma produciendo una luxación. La luxación traumática de la cadera como lesión aislada o asociada a fractura del acetábulo, la cabeza femoral, o ambas, es resultado de mecanismos de alta energía, principalmente de accidentes automovilísticos(Fernández Palomo, 2001). La mayoría de los 


\section{Tratamiento de luxación en pacientes con problemas óseos}

Vol. 3, núm. 2., (2019)

Paulina Elizabeth Duran Mora; Hernán David Toro Barrera; Mónica Carolina Calderón Moreira; Diana Galid San Martín Ajila; Fernanda Gisell García Reyes; Hugo Javier Saltos Giler

casos, este tipo de luxación está asociada a traumatismo de alta energía, y su reducción temprana reporta los mejores resultados a mediano y largo plazo (Haces, Legorreta, \& Osuna, 1997).

Este tipo de luxación aparece por grandes impactos que ha sufrido el cuerpo como son los accidentes de tránsito ocasionando que sean dos tipos que dependerá la posición de la extremidad al momento del impacto. Existen características mecánicas de las cuales dependerá la dirección de la luxación. La posición de la cadera, la dirección del vector fuerza y la propia anatomía del paciente son factores que influyen en el resultado de la lesión(Payo Rodríguez, Ibarzabal Gil, \& Rodríguez Merchán, 2006).

"La luxación posterior es 10 veces más frecuente que la anterior. Ésta se produce como resultado de una fuerza axial que actúa sobre el fémur con la cadera en flexión. La posición de la cabeza femoral al momento del impacto es determinante en la forma de la luxación, con flexión y aducción se favorece una luxación pura, mientras que con flexión y abducción se favorece fractura de la pared posterior del acetábulo previa a la luxación”. (Fernández Palomo, 2001, pág. 281)

La luxación anterior de la cadera es menos frecuente ya que se produce la abducción y extensión de la cadera. Es resultado de una posición en abducción y una fuerza en rotación externa; si esa misma fuerza se aplica con la cadera en flexión la luxación es inferior obturatriz; y si la fuerza se aplica en extensión, la luxación resultante es púbica(Fernández Palomo, 2001).

Ahora para poder determinar el diagnostico de este tipo de problema es necesario aplicar un criterio de evaluación clínica que pueda determinar las características de la enfermedad con el fin 


\section{Tratamiento de luxación en pacientes con problemas óseos}

Vol. 3, núm. 2., (2019)

Paulina Elizabeth Duran Mora; Hernán David Toro Barrera; Mónica Carolina Calderón Moreira;

Diana Galid San Martín Ajila; Fernanda Gisell García Reyes; Hugo Javier Saltos Giler

de aplicar un tratamiento que mejore la calidad de vida. Estos criterios se pueden visualizar en la Tabla 7.

Tabla 7. Criterios de evaluación clínica de la luxación traumática de cadera

\begin{tabular}{|l|l|}
\hline \multicolumn{1}{|c|}{ EXCELENTE } & \multicolumn{1}{c|}{ BUENO } \\
\hline $\begin{array}{l}\text { Sin dolor } \\
\text { Movilidad completa } \\
\text { Sin claudicación } \\
\text { Sin cambios en Rx }\end{array}$ & $\begin{array}{l}\text { Sin dolor } \\
75 \% \text { de movilidad } \\
\end{array}$ \\
\hline \multicolumn{1}{|c|}{ REGULAR } & $\begin{array}{l}\text { Ligera claudicación } \\
\text { Cambios mínimos en Rx }\end{array}$ \\
\hline $\begin{array}{l}\text { Uno o más de: } \\
\text { Dolor no incapacitante } \\
\text { Limitación de movimiento sin } \\
\text { deformidad en aducción } \\
\text { Cambios Rx moderadamente } \\
\text { severos }\end{array}$ & $\begin{array}{l}\text { Uno o más de: } \\
\text { Dolor incapacitante } \\
\text { Limitación marcada o } \\
\text { deformidad en aducción } \\
\text { Reluxación } \\
\text { Cambios Rx progresivos }\end{array}$ \\
\hline
\end{tabular}

Fuente: (Haces, Legorreta, \& Osuna, 1997)

Por lo tanto, el tratamiento para las luxaciones traumático de la cadera está dividido en varios tipos, como se observa en la Tabla 8 .

Tabla 8. Tratamientos de luxaciones traumáticas de cadera

\begin{tabular}{|c|c|}
\hline TRA? & $\begin{array}{l}\text { NTO } \\
\text { CCIONAL }\end{array}$ \\
\hline $\begin{array}{l}\text { Reducción Cerrada. } \\
\text { Es el tratamiento de la gran mayoría de las } \\
\text { luxaciones de cadera, considerándose una } \\
\text { emergencia incluso en aquellos casos con } \\
\text { fracturas conminutas de cabeza femoral o } \\
\text { con fractura acetabular. }\end{array}$ & $\begin{array}{l}\text { Luxaciòn sin fractura asociada. } \\
\text { Debemos evitar la carga precoz para } \\
\text { disminuir el riesgo de desarrollar una } \\
\text { necrosis avascular de cabeza femoral. De } \\
\text { cara al futuro, se está intentando usar la } \\
\text { RMN para saber en qué momento se debe }\end{array}$ \\
\hline
\end{tabular}




\section{Tratamiento de luxación en pacientes con problemas óseos}

Vol. 3, núm. 2., (2019)

Paulina Elizabeth Duran Mora; Hernán David Toro Barrera; Mónica Carolina Calderón Moreira; Diana Galid San Martín Ajila; Fernanda Gisell García Reyes; Hugo Javier Saltos Giler

Podemos considerar la reducción como tratamiento definitivo en las siguientes situaciones: cuando no hay fractura asociada ni incongruencia articular; cuando la fractura del muro posterior es clínicamente estable (se debe comprobar la estabilidad de la cadera bajo control radioscópico, realizando flexión y rotación interna de la cadera y ejerciendo presión en el eje del fémur para comprobar la estabilidad posterior; cualquier cambio observado en la relación de la cabeza femoral con el techo acetabular se puede considerar como subluxación y por lo tanto es una cadera inestable) y sin incongruencia articular (menor del $30 \%$ de la superficie articular).

Aquellas luxaciones irreducibles, las que muestran incongruencia articular o las que presentan una fractura asociada, pueden requerir una intervención quirúrgica.

\begin{tabular}{lllll}
\hline Reducción Abierta con & o & sin \\
Desbridamiento.
\end{tabular}

Está indicada en las siguientes situaciones: luxación irreducible, yatrogenia sobre el nervio ciático, reducción incongruente con fragmentos incarcerados, con partes blandas interpuestas o con fracturas de cabeza femoral Pipkin tipo I.

Luxaciones irreducibles: Requieren reducción abierta de forma urgente; aproximadamente del 2 al $15 \%$ de las luxaciones de cadera son irreducibles por métodos cerrados.

Reducciones con incongruencia articular: A diferencia de una cadera irreducible, esto no es una emergencia; ocurren por la presencia de un fragmento óseo o por interposición de tejidos blandos y deben ser extraídos. Incluyen: avulsiones de la cabeza femoral, fracturas de la cabeza femoral Pipkin tipo I, fragmentos libres del muro posterior o fragmentos osteocondrales de la cabeza femoral. permitir la carga para evitar la aparición de la necrosis avascular, aunque hasta este momento no se tienen evidencias claras.

Los pacientes en los que se redujo en las 6 primeras horas, realizarán reposo durante dos semanas, seguido de movilización pasiva, evitando los últimos grados de movilidad durante las 6-8 primeras semanas para permitir la cicatrización de la cápsula. Se puede permitir la carga de forma parcial a partir de la $2^{a}-3^{a}$ semana, hasta la carga total en la sexta semana. Para aquellos casos en los que la reducción se ha realizado después de las 6 primeras horas y aquellos pacientes en los que se precisó reducción abierta, se recomienda no permitir la carga total hasta las 8-12 semanas

\section{Luxación con fractura asociada.}

El tratamiento depende de la lesión asociada a la luxación. En las fracturas del muro posterior o de la cabeza femoral, se debe evitar la movilización activa hasta la $6^{a}$ semana; hasta ese momento se pueden realizar ejercicios de movilización pasiva. Se debe evitar la carga hasta la $10^{\mathrm{a}}-12^{\mathrm{a}}$ semana.

En aquellos casos en los que hay indicación de cirugía, pero no hay incongruencia articular tras la reducción, se debe inmovilizar con una tracción transesquelética femoral en discreta abducción para evitar la lesión cartilaginosa hasta que se realice la cirugía. Durante este tiempo es esencial mantener una correcta relación entre la cabeza del fémur y el acetábulo, siendo necesario un control radiográfico periódico.

En aquellas fracturas del muro posterior en las que no se ha podido explorar la estabilidad de la articulación, se debe 
Paulina Elizabeth Duran Mora; Hernán David Toro Barrera; Mónica Carolina Calderón Moreira; Diana Galid San Martín Ajila; Fernanda Gisell García Reyes; Hugo Javier Saltos Giler

\begin{tabular}{|c|c|}
\hline & $\begin{array}{l}\text { colocar una tracción transesquelética } \\
\text { femoral, con la cadera mínimamente } \\
\text { flexionada para asegurar la reducción, hasta } \\
\text { que se pueda realizar la exploración de la } \\
\text { estabilidad }\end{array}$ \\
\hline $\begin{array}{l}\text { Reducción Abierta y Fijación Interna. } \\
\text { Sólo está indicada para algunos casos de } \\
\text { fractura luxación. } \\
\text { Fracturas del muro posterior: que afecten a } \\
\text { más del } 30 \% \text { de la superficie articular, ya } \\
\text { que estas caderas se consideran inestables } \\
\text { debido a la extensión de la fractura; o las } \\
\text { que afecten a menos del } 30 \% \text { de la } \\
\text { superficie articular, pero que provocan una } \\
\text { inestabilidad en la cadera (tipo III de } \\
\text { Stewart y Milford) } \\
\text { Fracturas de cuello femoral: En pacientes } \\
\text { jóvenes: requiere la reducción abierta y } \\
\text { osteosíntesis precoz de la fractura; si la } \\
\text { fractura no está desplazada, la osteosíntesis } \\
\text { puede realizarse previamente a la reducción; } \\
\text { si la fractura está desplazada, la reducción } \\
\text { de la cabeza facilitará la reducción de la } \\
\text { fractura. Por otro lado, las fracturas de } \\
\text { cuello femoral en pacientes de edad } \\
\text { avanzada, el tratamiento de elección es la } \\
\text { artroplastia o hemiartroplastia de } \\
\text { sustitución. } \\
\text { Fracturas de cabeza femoral: En las } \\
\text { fracturas Pipkin tipo II, si al realizar la } \\
\text { reducción de la luxación el fragmento no } \\
\text { está reducido correctamente, se debe } \\
\text { realizar osteosíntesis. } \\
\text { Impactaciones de la cabeza femoral: Deben } \\
\text { intervenirse cuando se afecta una superficie } \\
\text { articular mayor de } 2 \text { cm2; se realiza una } \\
\text { elevación del fragmento hundido y aporte } \\
\text { de injerto. }\end{array}$ & \\
\hline
\end{tabular}

Fuente: (Payo Rodríguez, Ibarzabal Gil, \& Rodríguez Merchán, 2006). 


\section{Tratamiento de luxación en pacientes con problemas óseos}

Vol. 3, núm. 2., (2019)

Paulina Elizabeth Duran Mora; Hernán David Toro Barrera; Mónica Carolina Calderón Moreira; Diana Galid San Martín Ajila; Fernanda Gisell García Reyes; Hugo Javier Saltos Giler

\section{Discusión y conclusiones}

Una de las estructuras más importantes dentro del cuerpo humano es la estructura ósea. Esta estructura esquelética sostiene a todos los órganos como también puede producir el equilibrio y la estabilidad física en el individuo. Los huesos son órganos que crecen y se regeneran en casi todo el tiempo de vida, los cuales garantizan el comportamiento mecánico y permite que se puedan distribuir las cargas físicas en toda la estructura. Esta regeneración constante es producida por células que tienen como los osteoblastos, que son encargados de la formación de la matriz ósea, los osteocitos los cuales participan en las funciones metabolicas y los osteoclastos que participa en la función de la resorción ósea. Este tipo de característica de los huesos se realiza hasta los 35 años de edad, después de los 40 años empieza un proceso degenerativo y con ello el sistema esquelético empieza a debilitar en sus propiedades mecánicas.

Una de las consecuencias de la vejez es que continuamente existen problemas en los huesos y articulaciones que no permiten tener una mejor salud en esa edad avanzada. Es decir, que las personas de la tercera edad son más propensas en sufrir problemas óseos. Dentro de estos problemas óseos están los más importantes las cuales son la artrosis y la osteoporosis. La primera afecta las articulaciones de los huesos porque hay pérdida de los cartílagos presentes en cualquier zona, específicamente en las rodillas, manos y cadera. La segunda se representa por la pérdida de la masa ósea de los huesos permitiendo que las características mecánicas disminuyan y sean más propensas a sufrir fracturas en las rodillas, hombros, manos y cadera. Una de las características más vulnerables en las personas de la tercera edad es el sufrimiento y dolor en las caderas es por ello que esta investigación se centra en este tipo de zona. Cuando es la artrosis en 


\section{Tratamiento de luxación en pacientes con problemas óseos}

Vol. 3, núm. 2., (2019)

Paulina Elizabeth Duran Mora; Hernán David Toro Barrera; Mónica Carolina Calderón Moreira;

Diana Galid San Martín Ajila; Fernanda Gisell García Reyes; Hugo Javier Saltos Giler

la cadera se manifiesta como perdida de los cartílagos presentes entre cada vertebra y cuando es la osteoporosis es por la pérdida de masa ósea de las vértebras de la columna.

Las luxaciones se manifiestan como un desplazamiento del hueso de la articulación que lo mantiene unido. Estas se presentan en personas jóvenes y en aquellas que realizan trabajos forzosos donde se pueda perder el equilibrio esquelético y permita un movimiento brusco para que le hueso salga de su posición. Estas luxaciones se presentan con mayor frecuencia en personas con problemas óseos, por lo que las personas en avanzada edad tienen mayor probabilidad de sufrirlas. Como el problema de cadera es una de las enfermedades más frecuentes en este tipo de personas y más porque son propensos a sufrir artrosis y osteoporosis entonces es necesario poder determinar un diagnostico que permita mejorar esa calidad de vida. Estos tratamientos se basarán si son con reducción cerrada, que es la más común en su tipo, reducción abierta con o sin desbridamiento y reducción abierta y fijación interna. También existe tratamiento postreduccinal basado en luxaciones con o sin fractura asociada.

\section{Bibliografía.}

Aguilar Caballero, I., \& Galbez García de Aguilar, T. (1976). Tratado práctico de medicina moderna. California - Estados Unidos: Ediciones Interamericanas.

Aresti, N. (2016). Artrosis de cadera. IntraMed, 1-5.

Carmona Ortells, L. (2010). Epidemiología de la artrosis. En J. Monfort, Artrosis Fisiopatología, diagnóstico y tratamiento (págs. 3-17). Mdrid - España: Sociedad Española de Reumatología. Editorial Médica Panamericana.

De Reumatología, S. C. (2007). Guías de diagnóstico, prevención y tratamiento de la osteoporosis. Rev. chil. reumatol, 23(2), 45-84.

Dotor, S. R., \& Domínguez Hernández, V. M. (2002). Alternativas para obtener un injerto óseo. Acta Ortopédica Mexicana 16.4, 225-231. 


\section{Tratamiento de luxación en pacientes con problemas óseos}

Vol. 3, núm. 2., (2019)

Paulina Elizabeth Duran Mora; Hernán David Toro Barrera; Mónica Carolina Calderón Moreira;

Diana Galid San Martín Ajila; Fernanda Gisell García Reyes; Hugo Javier Saltos Giler

Estrada, A. F., Gallard, L. E., Fernandez, C. B., \& Martinez, O. S. (1998). Osteoporosis, epidemia del año 2000. Revisión bibliográfica. Revista Información Científica, 19(3), 18.

Fernández Palomo, L. J. (2001). Nueva clasificación para la luxación traumática de la cadera. Acta Ortopédica Mexicana, 15(6), 280-287.

FORRIOL, F. (2005). Los sustitutos óseos y sus posibilidades actuales. Rev Asoc Argent Ortop Traumatol, 70, 82-93.

Gabrielli, L., \& Rodríguez, J. (2004). Osteoporosis. OMS.

Giménez, C. A., Zafra, G. A., \& González, M. D. (2006). OSTEOPOROSIS Y OSTEOMALACIA. En TRATADO de GERIATRÍA para residentes. Retrieved JULIO, $22,2013$.

Gracia-Marco, L., Vicente-Rodríguez, G., Valtueña, J., Rey-López, J. P., Díaz Martínez, A. E., Mesana, M. I., . . . Moreno, L. A. (2009). Marcadores del metabolismo óseo en adolescentes españoles. Estudio HELENA. Trauma 21.1, 33-38.

Gutiérrez, B. V., \& Ekdahl, G. M. (2014). Artrosis de Hombro. Revista Médica Clínica Las Condes, 25(5), 732-737.

Haces, F., Legorreta, J. G., \& Osuna, G. (1997). Luxación Traumática de cadera en niños, Reporte de 10 casos. Revista Mexicana de Ortopedia Pediátrica, 1(1), 27-31.

Hermoso de Mendoza, M. T. (2003). Clasificación de la osteoporosis: Factores de riesgo. Clínica y diagnóstico diferencial. In Anales del sistema sanitario de Navarra. Vol. 26. Gobierno de Navarra. Departamento de Salud., 29-52.

INSHT. (21 de Mayo de 2019). NTP 546: Primeros auxilios: fracturas, luxaciones y esguinces. Obtenido de http://www.insht.es/InshtWeb/Contenidos/Documentacion/FichasTecnicas/NTP/Ficheros $1501 \mathrm{a} 60$

López, F. (2006). Artrosis. Revista clínica electrónica en atención primaria, (11), 1-4.

Mamani Conde, E. Y. (2018). La sensibilización central en artrosis de rodilla. Evaluación diagnóstico y tratamiento. Lima - Perú: Trabajo de Grado - Universidad Inca Garcilaso De La Vega.

Martínez, Y. I., Molina, J. J., Consuegra, A. M., Vázquez, H. J., \& Fernández, F. R. (2006). La osteoporosis: una epidemia silenciosa. Medisur: Revista Electrónica de las Ciencias Médicas en Cienfuegos, 4(1), 85-91. 


\section{Tratamiento de luxación en pacientes con problemas óseos}

Vol. 3, núm. 2., (2019)

Paulina Elizabeth Duran Mora; Hernán David Toro Barrera; Mónica Carolina Calderón Moreira;

Diana Galid San Martín Ajila; Fernanda Gisell García Reyes; Hugo Javier Saltos Giler

Miralles, R. (2010). 7.4. Fisioterapia en el tratamiento de las fracturas y las luxaciones. Tarragona: Centre de Cooperació al Desenvolupament, URV Solidaria. Universitat Rovira i Virgili.

Morgado, I., Pérez, A. C., Moguel, M., Pérez-Bustamante, F. J., \& Torres, L. M. (2005). Guía de manejo clínico de la artrosis de cadera y rodilla. Revista de la Sociedad Española del Dolor, 12(5), 289-302.

Padrón, M. J., González, L. P., de León, R. P., Fiallo, I. R., \& Kim, S. E. (2006). Osteoporosis:¿ Un problema de salud prevenible? Revista Médica Electrónica, 28(5), 452-459.

Payo Rodríguez, J., Ibarzabal Gil, A., \& Rodríguez Merchán, E. C. (2006). Luxación traumática de cadera. Patología del aparato locomotor, 4(3), 167-178.

Perrot, S. (2016). Mecanismos neuropáticos y Síntomas en el Dolor Articular: Impacto en el Diagnostico y en el tratamiento. FACT SHEET No. 12, 1-4.

Vera Velandia, A., \& Tovar Pérez, A. (2008). Estudio computacional de las microgrietas, la apoptosis y el envejecimiento en el remodelamiento óseo. Revista Ingeniería Biomédica, 2(4), 67-77.

Zárate, A., Basurto, L., \& Saucedo, R. (2010). El tejido óseo se incorpora a la constelación hormonal. Revista Médica del Instituto Mexicano del Seguro Social, 48(1), 1-5. 